\title{
INTRODUCCION AL ESTUDIO DE LOS HONGOS SUPERIORES V.
}

(Introduction to the study of higher fungi V.)

WALDO LAZO

Departamento de Ciencias Ecológicas, Facultad de Ciencias, Universidad de Chile, Casilla 653, Santiago, Chile.

Palabras clave: Basidiomycetes, hongos comestibles, hongos venenosos. Key words: Basidiomycetes, edible fungi, poisonous fungi.

\section{RESUMEN}

Se presenta ilustraciones y descripciones de 12 Basidiomycetes comunes en Chile. Seis de ellos comestibles: Grifola gargal, Ramaria flava, Pleurotus ostreatus, Agrocybe aegerita, Agaricus arvensis, Agaricus campestris. Seis de ellos venenosos: Amanita muscaria, Lepiota cristata, Mycena pura, Paxillus involutus, Psilocybe zapotecorum, Scleroderma flavidum.

Continuando con el propósito de presentar ilustraciones en color de especies fúngicas comunes en Chile (Lazo, 1984, 1996, 1998) se incluye en esta publicación las ilustraciones de los basidiomas de seis hongos comestibles $\mathrm{y}$ de seis hongos venenosos. Al igual que en las publicaciones antes citadas, estos textos e ilustraciones forman parte de "Hongos de Chile. Atlas Micológịco" (Lazo, inédito).

Se recomienda, una vez más, no comer nunca setas crudas debido a la posible existencia de sustancias cancerígenas que se destruyen mediante la cocción.

1.- Grifola gargal Singer, Beih. Nova Hedwgia 29:381-382. 1969.

BASIDIOMA de 250 o más $\mathrm{mm}$ de anchura, multipileado, (píleos) imbricados. PILEO parduzco a oliváceo-parduzco, más bien delgado. POROS blancos, decurrentes. CONTEXTURA blanca, carnosa. SABOR ligeramente ácido astringente. OLOR fúngico tenue. ESPORADA blanca. ESPORAS de 7-7-x 5.2-5.5 $\mu \mathrm{m}$, hialinas elipsoides. BASIDIOS de 24-36 x 7-7-.5 $\mu \mathrm{m}$, claviformes, tetrasporados. No hay cistidios.

HABITAT : sobre madera (troncos, tocones, ramas). DISTRIBUCION : desde Chillán hasta Aysén. NOM-

\section{SUMMARY}

Description and color ilustrations of 12 common Chilean Basidiomycetes are presented. Six of them edible: Grifola gargal, Ramaria flava, Pleurotus ostreatus Agrocybe aegerita, Agaricus arvensis, Agaricus campestris. Six of them poisonous: Amanita muscaria, Lepiota cristata, Mycena pura, Paxillus involutus, Psilocybe zapotecorum, Scleroderma flavidum.

BRE VULGAR: "Gargal". Comestible de buen sabor.

E.I.: precordillera de Chillán. Mayo, 1999 (Foto gentileza Srta. Mónica Cortez).

2.- Ramaria flava (Fr.) Quél. Fl.Myc.466-1888

BASIDIOMA de 120-200 x 80-160 mm, amarillo ocráceo, ramificado. ESTIPITE de 50-80 × 40-50 mm, blanquizco en la parte basal. CONTEXTURA amarillenta, frágil. SABOR tenue. ESPORADA parda. ESPORAS de 10-12 x $5 \mu \mathrm{m}$, ocráceas, verruculosas, elongado-elipsoides, gutuladas.

HABITAT: en el suelo. DISTRIBUCION: AMPLIA. Desde Chillán hasta Aysen. NOMBRE VULGAR: "Changle". Comestible. De Chillán al sur se lo puede comprar en ferias y mercados durante el otoño.

A Ramaria valdiviana Singer, a otras Ramaria spp. y a otras Clavariaceas comestibles también las llaman "changle".

E.I.: precordillera de Chillán. Mayo 1999 (Foto gentileza de Srta. Mónica Cortéz)

3.- Agrocybe aegerita (Brig.) Sing., Schweiz. Z. Pilzk. 17:21. 1939.

PILEO de 50-140 mm. de diámetro, de color pardusco cuero a castaño, blanquecino hacia los bordes, 
convexo al principio finalmente plano, seco, liso, con el margen incurvado.LAMELAS al principio pálidas despues pardo-canela, adnadas a algo decurrentes, congregadas. ESTIPITE de 60-150 x 15-30 mm, blanquizco, cilindráceo, atenuado hacia la base, duro, fibriloso, a veces estriado. ANILLO blanco, persistente, membranáceo, grande, generalmente muestra la impronta de la esporada en su cara superior. CONTEXTURA blanquizca, carnosa. OLOR agradable (con cierta semejanza al de una antigua pipa vinera). SABOR agradable (parecido al de la avellana) ESPORADA pardo tabaco. ESPORAS de 8-10 x 5-6 $\mu \mathrm{m}$, pardas, lisas, elipsoides. CISTIDIOS claviformes. PILEIPELLIS himeniforme con células isodiamétricas. HIFAS con FIBULAS.

HABITAT: se desarrolla sobre sauces o álamos vivos o muertos especialmente durante el otoño, e invierno. Puede cultivársela artificialmente en estas maderas durante todo el año.DISTRIBUCIÓN: amplia, Chile central y austral. Comestible de buen sabor.

\section{Agaricus arvensis Schff. ex Fr., Syst Mycol.} 1:281. 1821.

PILEO de 80-150 mm de diámetro, blanco, al envejecer amarillento a ocre pálido, convexo cuando joven después convexo aplanado, liso o flocoso o con escamas, seco. LAMELAS libres, de color gris pálido, después rosáceas, finalmente pardo purpúreas. ESTIPITE de 80-130 x 15 -25 $\mathrm{mm}$, concoloro con el píleo, carnoso, con la base algo engrosada, flocoso bajo el anillo. ANILLO bien desarrollado, persistente. CONTEXTURA (carne) gruesa, blanca, ocrácea al envejecer, SABOR dulzón, grato. OLOR anisado. ESPORADA pardo-purpúrea. ESPORAS de 7-8 $55 \mu \mathrm{m}$, pardo purpúreas, ovales.

HABITAT: en el suelo, en prados, jardines, potreros. DISTRIBUCION: amplia; Chile central y austral. Comestible de buen sabor. No comer crudo.

\section{Agaricus campestris L. ex Fr., Syst. Mycol.} $1: 281.1821$.

PILEO de 50-100 o más mm de diámetro, blanquizco a ocráceo, con escamas parduzcas, convexo aplanado, sedoso, fibriloso. LAMELAS libres, rosadas al principio pardo-purpúreas al envejecer. ESTIPITE de 30-70 x 10-13 mm, blanquizco, liso aunque algo escamoso bajo el anillo, atenuado. ANILLO blanquizco, pequeño, frágil, efimero. CONTEXTURA blanca al principio, después rosada. OLOR fúngico. SABOR tenue. ESPORADA pardo-purpúrea. ESPORAS de 7-8- $x$ 4-5 $\mu \mathrm{m}$., pardo purpúreas, ovoides.

HABITAT:en el suelo, en prados y potreros. DISTRIBUCIÓN: amplia, Chile central y austral. Comestible de muy buen sabor. No comer crudo.
6.- Pleurotus ostreatus (Jacq.ex.Fr.) Quel., Champ.Jura Vosg. 1:112. 1872

PILEO de 70-160 mm. de ancho, de color pizarrapardo intenso cuando nuevo, más pálido al envejecer, conchoide a sub-dimidiado, algo convexo, carnoso, margen delgado. ESTIPITE de $20 \times 20 \mathrm{~mm}$, a veces menor, a veces inexistente. LAMELAS blancas, amarillentas al envejecer, anchas, anastomosantes en la base. CONTEXTURA (carne) blanca. SABOR agradable. OLOR fúngico. ESPORADA lila pálido. ESPORAS de 8-11 x 5-6 $\mu \mathrm{m}$, elípticas, gutuladas.

HABITAT: sobre troncos y ramas de diferentes especies arbóreas. DISTRIBUCIÓN: amplia, Chile central y austral. También se lo cultiva en criaderos. Comestible.

E.I.: precordillera de Chillán. Mayo 1999. (foto gentileza de Srta. Mónica Cortéz).

\section{Amanita muscaria (L. ex Fr.) Hooker, Fl,} Scot. 2:19. 1821

PILEO de 100-120 mm. de diámetro, rojo bermellón cuando joven, rojo anaranjado muchas veces moteado de amarillo al envejecer, semigloboso a veces ligeramente cóncavo, cubierto de múltiples escamas flocosas por lo general de color blanco, efimeras. Margen estriado algo incurvado. LAMELAS blancas, libres desiguales. ESTIPITE de 120-220 x 15-25 mm, blanco amarillento, bulboso, con un ANILLO bien desarrollado, blanco, escamoso. VOLVA blanca, fragmentada en estructuras como escamas o verrugas dispuestas en anillos concéntricos. CONTEXTURA blanca, algo rojiza bajo el pileipellis. SABOR a nueces. OLOR aromático "sui generis". ESPORADA blanca. ESPORAS de 9-11 x 6-8 $\mu \mathrm{m}$, ovoides, lisas, hialinas.

HABITAT: en el suelo en bosques de coníferas, abedules, eucaliptos. DISTRIBUCIÓN: Europa, América, Chile(Valdivia). Tóxica .

8. Mycena pura (Fr.) Quél., Jura et Vosg., :103. 1871.

PILEO de 20-40 mm de diámetro, violáceo, amatista claro, rosado, campanulado a convexo, finalmente expandido, obtusamente umbonado, húmedo, estriado en el margen. LAMELAS lila pálido, adnadas, sinuadas, moderadamente anchas, ventricosas. ESTIPITE de 50-100 x 2-6 $\mathrm{mm}$, concoloro con el píleo, cilíndrico, finamente pruinado, glabro, hueco, más o menos velloso en la base. ESPORAS de 6.8-9 $\times$ 4-4.5 $\mu \mathrm{m}$, hialinas, elipsoides, cilíndricas, débilmente amiloides, lisas. BASIDIOS de 20-28 x 8.2-10.3 $\mu$, tetrasporados. CISTIDIOS escasos, clavado-cilíndricos. TRAMA del píleo, himenóforo y estípite fuertemente amiloides. Todas las HIFAS con FIBULAS.

HABITAT: se desarrolla en los prados, sobre made- 

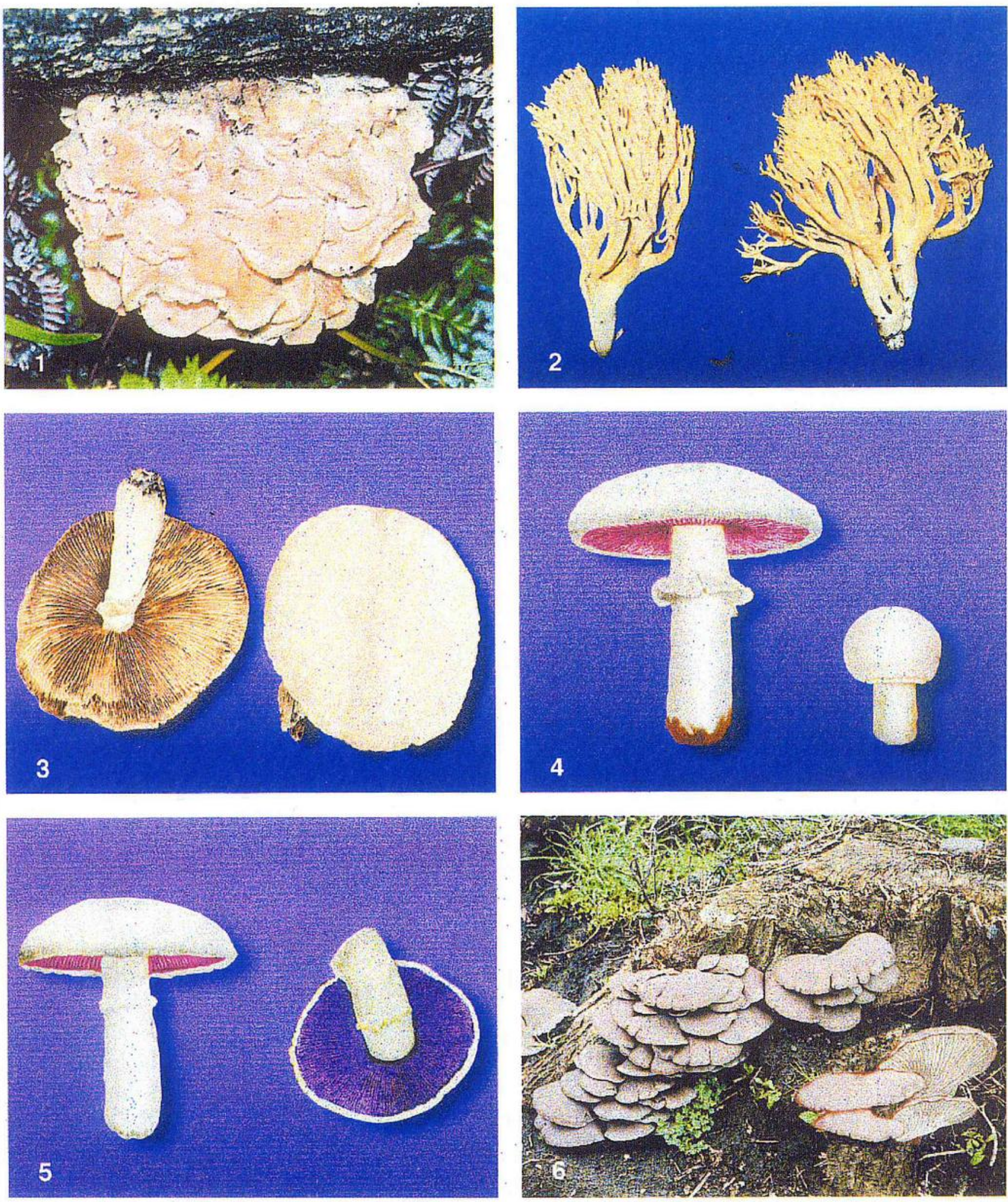

HONGOS COMESTIBLES

Figuras (1-6): 1. Grifola gargal 2. Ramaria flava 3. Agrocybe aegerita 4. Agaricus arvenis 5. Agaricus campestris 6. Pleurotus ostreatus 

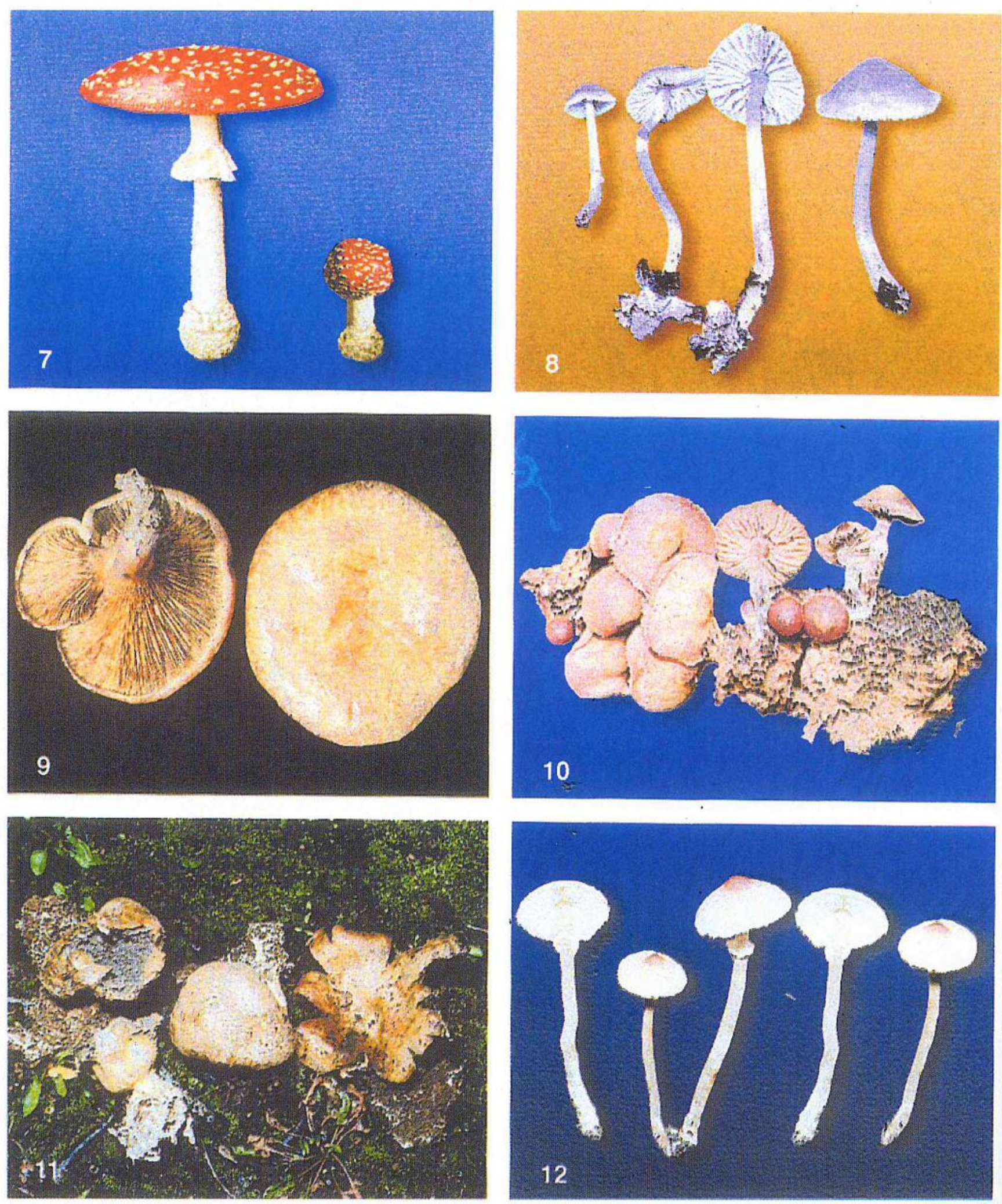

\section{HONGOS VENENOSOS}

Figuras (7-12): 7. Amantia muscaria 8. Mycena pura 9. Paxillus involutus 10. Psilocybe zapotecorum 11. Scleroderma flavidum 12. Lepiota cristata 
ras en descomposición. DISTRIBUCIÓN: amplia. Tóxica.

\section{9.- Paxillus involutus (Batsch. ex Fr.) Fr., Epicr.} Syst Mycol. 317. 1838.

PILEO de 40-90 mm de diámetro, pardo ocráceo pardo rojizo, convexo cuando nuevo, con posterioridad expandido deprimido, cuando nuevo presenta el márgen incurvado, viscido al mojarlo, brillante cuando seco. LAMELAS pardusco amarillas, decurrentes a veces anastomosantes formando poros cerca del estípite. ESTIPITE de 40-60 x 10-25 mm, pardusco, sólido, con puntos, parejo a algo engrosado en la base. ESPORADA parda. ESPORAS de 8-9 × 5-6 $\mu \mathrm{m}$, parduscas, lisas, elípticas. CISTIDIOS de 60-75 x 8-14 $\mu \mathrm{m}$, lanceolados.

HABITAT: en el suelo entre los restos vegetales. DISTRIBUCIÓN: muy amplia. Tóxica.

10.- Psilocybe zapotecorum Heim. emend. Guzmán, Rev. Mycol. (París) 22: 77. 1957.

PILEO de $12 \mathrm{~mm}$ de diámetro, pardo amarillento, umbonado, higrófano. LAMELAS negruzcas, adnadas. ESTIPITE de $15-18 \times 3 \mathrm{~mm}$, pardo obscuro a, pardo castaño, escamoso fibriloso, blanco en la base. OLOR ureico penetrante. SABOR dulzón, ESPORAS de 7-7.2 x 4-4.3 $\mu \mathrm{m}$, pardo amarillentas lentiformes. BASIDIOS de 15-23 x 5-7 $\mu \mathrm{m}$, hialinos, claviformes. QUEILOCISTIDIOS de $19-28 \times 6-$ $12 \mu \mathrm{m}$, fusiforme-ampuláceos. CRISOCISTIDIOS de 19-28 $\mathrm{x}$ 6-12 $\mu \mathrm{m}$, vesiculosos, pedicelados o claviformes.

HABITAT: sobre estiércol vacuno. DISTRIBUCIÓN: amplia. Tóxica.

11. Scleroderma flavidum Ell. et Ev., Mycol., 1:88. 1885.

GASTROCARPO de 20-50 mm de ancho, globoso deprimido a subgloboso, superficial o parcialmente enterrado, firme, con una base estipitoide y bien desarrollada. PERIDIO amarillo ante a color paja, firme y resistente, deshisce por diversos lóbulos, con la parte superior generalmente areolada. GLEBA olivácea a amarillenta y que usualmente desaparece. PLACAS de la trama amarillas y a veces persistentes. ESPORAS de 9-13 $\mu \mathrm{m}$, ferruginoso pardas, globosas, marcadamente equinadas, con espinas de $0.6 \mu \mathrm{m}$ de longitud. CAPILICIO de 4.4-6.5 $\mu \mathrm{m}$ de ancho, hialino, liso, escasamente septado.

HABITAT: en el suelo en los márgenes de un bosque de Nothofagus.DISTRIBUCIÓN: amplia.Tóxica

12.- Lepiota cristata (Alb. et Schweín. ex Fr. ) Kummer, Führer Pilzk. 137. 1871.

PILEO de 15-22 mm de diámetro, de color rojizo pardo ladrillo sobre fondo blanco, convexo, escamoso. LAMELAS blancas, juntas, libres. ESTIPITE de 30-55 × 4-6 $\mathrm{mm}$, blanco, algo escamoso abajo, blanquizco a levemente pardo, con un anillo bien formado que posee un margen membranoso rojizo, glabro por debajo del anillo. CONTEXTURA más o menos frágil. ESPORADA blanca. ESPORAS de 7-7.5 $\times 3 \mu \mathrm{m}$, hialinas, marcadamente "eperonnées", lisas. BASIDIOS de 17-18.5 x 6-6.5 $\mu \mathrm{m}$, tetrasporados. QUEILOCISTIDIOS de $21 \times 7-11 \mu \mathrm{m}$, hialinos o ligeramente opacos, claviformes, la mayoría largo-pedicelados, algunos con apéndices filamentosos. HIFAS con FIBULAS.

HABITAT: se desarrolla en el suelo entre las hojas, secas y restos vegetales, dentro de los bosques. DISTRIBUCIÓN: amplia. Tóxica

\section{REFERENCIAS}

Garrido, N. (1985). Index Agaricalium Chilensium. J. Cramer. Vaduz.

Lazo, W. (1984). Introducción al estudio de los hongos superiores III. Bol.Micol. 2: 27-66

Lazo, W. (1996). Algunos Ascomycetes y Basidiomycetes interesantes de Chile. Bol.Micol. 11: 99-102

Lazo, W. (1998). Introducción al estudio de los hongos superiores. IV. Bol. Micol. 13: 71-75
Singer, R. (1969). Micoflora Australis. Beih. Nova Hedwigia 29:1-406

Spegazzini, C. (1910). Fungi Chilensis. Contribución al estudio de los hongos chilenos. Librería Nacional. Buenos Aires.

Cs. $25: 1-124$ (1921). Mycetes Chilensis. Bol. Acad. Nat. 